\title{
OPEN Contrast enhanced magnetic resonance imaging highlights neurovasculature changes following experimental traumatic brain injury in the rat
}

David K. Wright ${ }^{1,5}$, Jamie N. Mayo ${ }^{2,3,4,5}$, Mujun Sun ${ }^{1,2}$, Terence J. O'Brien ${ }^{1,2} \&$ Sandy R. Shultz ${ }^{1,2 \bowtie}$

Neurovascular injury has been proposed as a universal pathological hallmark of traumatic brain injury (TBI) with molecular markers of angiogenesis and endothelial function associated with injury severity and morbidity. Sex differences in the neurovasculature response post-TBI may contribute to the differences seen in how males and females respond to injury. Steady-state contrast enhanced magnetic resonance imaging (SSCE-MRI) can be used to non-invasively assess the neurovasculature and may be a useful tool in understanding and predicting outcomes post-TBI. Here we used SSCE-MRI to investigate the neurovasculature of male and female rats at $48 \mathrm{~h}$ after an experimental TBI, and how these changes related to neuromotor function at 1-week post-TBI. In addition to TBI induced changes, we found that female rats had greater vessel density, greater cerebral blood volumes and performed better on a neuromotor task than their male counterparts. These results suggest that acute post-TBI cerebrovascular function is worse in males, and that this may contribute to the greater functional deficits observed post-injury. Furthermore, these results highlight the potential of SSCEMRI to provide insights into the cerebral microvasculature post-TBI. Future studies, incorporating both males and females, are warranted to investigate the evolution of these changes and the underlying mechanisms.

Neurovascular injury has been proposed as a universal pathological hallmark of traumatic brain injury (TBI) ${ }^{1}$. Changes in microvascular density have been detected acutely after TBI and are thought to contribute to early pathogenesis and reflect the extent of neurological damage $e^{2,3}$. Microvascular density in the weeks following TBI has also been associated with recovery ${ }^{3,4}$, and alterations in cerebral blood flow can persist for months post-injury and may contribute to both recovery and continued pathology $y^{3,5}$. Molecular markers of new blood vessel growth, angiogenesis, and endothelial function have also been associated with injury severity and morbidity after TBI ${ }^{6}$.

There is increasing evidence that males and females differ in various outcomes after TBI, underscoring the notion that there are biological differences between the sexes that account for this ${ }^{7-11}$. Of particular relevance, there is initial evidence suggesting sex differences in neurovascular measures post-TBI, and that these measures correlate with neurological recovery ${ }^{3,12}$. For example, a recent study found that there were similar vessel losses in both males and females at 1-day post-injury, but revascularization was greater and more complex in the ipsilateral cortex of males at 7 days post-injury ${ }^{4}$. Given the role of the neurovasculature in both acute and long-term consequences of TBI, and the association of neurovascular response with TBI severity and morbidity, acute vascular density changes could be an important sex specific prognostic indicator of patient recovery.

Identifying clinically applicable biomarkers that are sensitive to sex differences would aid in the development of personalized therapeutic approaches for the treatment of TBI. Steady-state contrast enhanced

${ }^{1}$ Department of Neuroscience, Central Clinical School, Monash University, The Alfred Centre, 99 Commercial Road, Melbourne, VIC 3004, Australia. '2Department of Medicine, Royal Melbourne Hospital, University of Melbourne, Parkville 3052, Australia. ${ }^{3}$ DDEAS 2.0 Center of Innovation, VA Salt Lake City Health Care System, Salt Lake City, UT, USA. " Division of Epidemiology, Department of Medicine, University of Utah School of Medicine, Salt Lake City, UT, USA. ${ }^{5}$ These authors contributed equally: David K. Wright and Jamie N. Mayo. ${ }^{\square}$ email: sandy.shultz@ monash.edu 


\begin{tabular}{|l|l|l|l|l|}
\hline Cohort & Male+Sham & Male+ TBI & Female+Sham & Female+ TBI \\
\hline $\mathrm{n}$ & 11 & 15 & 11 & 14 \\
\hline Weight $(\mathrm{g})$ & $463.7 \pm 62.0$ & $450.1 \pm 41.6$ & $291.6 \pm 9.4$ & $302.5 \pm 25.9$ \\
\hline Pain reflex $(\mathrm{s})$ & 0 & $269.5 \pm 94.8$ & 0 & $355.3 \pm 126.6$ \\
\hline Self-righting $(\mathrm{s})$ & $292.1 \pm 68.9$ & $844.6 \pm 266.4$ & $154.6 \pm 54.7$ & $935.0 \pm 276.3$ \\
\hline
\end{tabular}

Table 1. Acute injury severity measures and cohort numbers. Male rats weighed significantly greater than female rats at the time of injury $(p<0.0001)$. Rats given a TBI had significantly longer pain reflex and selfrighting times compared to sham rats $(p<0.0001)$. Numbers represent mean \pm SD.

magnetic resonance imaging (SSCE-MRI) is a relatively recent imaging method that can be used to assess the neurovasculature ${ }^{13}$. SSCE-MRI uses an iron-based contrast agent and allows the estimation of cerebral blood volume $(\mathrm{CBV})$ changes in the microvasculature, as well as changes in vessel density and size. Importantly, SSCE-MRI derived measurements of vessel density and size have a strong positive correlation with histological measurements ${ }^{13,14}$, and the technique has been utilized to monitor the treatment effect of drugs targeting angiogenesis ${ }^{15}$.

While SCCE-MRI provides a means to investigate sex-specific neurovascular changes after TBI, its utility in this context has yet to be explored. Furthermore, it is unknown how SSCE-MRI measures relate to recovery after TBI. Therefore, this study used a pre-clinical rodent model of TBI to test the hypothesis that SSCE-MRI neurovasculature measurements could identify sex differences post-TBI and examined how SSCE-MRI measures related to functional outcomes.

\section{Materials and methods}

Subjects. 51 adult male $(n=26)$ and female $(n=25)$ Long-Evans rats were purchased from Monash animal research services (Melbourne, Australia). All rats were 10-15 weeks of age at the time of surgery with male rats significantly heavier than female rats (Table 1). All females were in diestrus at the time of injury. Following surgery, rats were housed individually under a $12 \mathrm{~h} / 12 \mathrm{~h}$ light/dark cycle with ad libitum access to water and food for the duration of the study. All experimental procedures were approved by The University of Melbourne and The Florey Institute of Neuroscience and Mental Health animal ethics committees, and complied with the guidelines of the Australian Code of Practice for the Care and Use of Animals for Scientific Purposes.

Experimental design. Rats were randomly assigned to either sham ( $\mathrm{n}=11$ male; $\mathrm{n}=11$ female) or lateral fluid percussion injury (LFPI) groups ( $\mathrm{n}=15$ male; $\mathrm{n}=14$ female) and underwent 48 -h SSCE-MRI ( $\mathrm{n}=12$ male, 6 LFPI; $n=12$ female, 6 LFPI) and/or 1-week behaviour ( $n=23$ male, 14 LFPI; $n=19$ female, 10 LFPI). Animal numbers were determined based on earlier SSCE-MRI and behaviour studies ${ }^{16,17}$ and animals were randomly assigned to each group.

Lateral fluid percussion injury (LFPI). Sham and LFPIs were performed as described previously ${ }^{18-20}$. Briefly, rats were anaesthetized and their head was immobilized using a standard stereotaxic device with anesthesia maintained using $2 \%$ Isoflurane in $500 \mathrm{ml} / \mathrm{min}$ oxygen via a nosecone. The dura matter of the left hemisphere was exposed by a craniectomy $(5 \mathrm{~mm}$ diameter) centered $-3.0 \mathrm{~mm}$ posterior and $4.0 \mathrm{~mm}$ lateral to bregma. A hollow injury cap was fixed to the skull, anesthesia was terminated, and the injury cap connected to the fluid percussion device. At the first sign of hind-limb withdrawal to a toe-pinch, a fluid percussion pulse of 3 atmospheres was delivered to the intact dura. Pain (i.e. hind-limb withdrawal to a toe-pinch) and self-righting reflexes were recorded immediately post-injury (see Table 1$)^{21}$. Once spontaneous breathing had resumed the injury cap was removed and the incisions sutured. Sham rats underwent the same procedure, including craniectomy, but no fluid pulse was given.

Steady-state contrast enhanced MRI (SSCE-MRI). SSCE-MRI was performed using a $4.7 \mathrm{~T}$ MRI at $48 \mathrm{~h}$ post-injury. Baseline $\mathrm{T}_{2}{ }^{*}$-weighted and $\mathrm{T}_{2}$-weighted images were acquired over the same 12 slices with a field of view $=38.4 \times 38.4 \mathrm{~mm}^{2}$ and slice thickness $=1 \mathrm{~mm}$. $\mathrm{T}_{2}{ }^{*}$-weighted images were acquired with a FLASH sequence with repetition time (TR)/echo time $(\mathrm{TE})=400 / 10 \mathrm{~ms}$; number of excitations $(\mathrm{NEX})=6$; and flip angle $=20^{\circ} . T_{2}$-weighted images were acquired with a RARE sequence and TR/TE $\mathrm{eff}=2300 / 65 \mathrm{~ms}$; RARE factor $=8$; and $\mathrm{NEX}=8$. Following the acquisition of these preliminary images, a bolus of $15 \mathrm{mg} / \mathrm{kg}$ of ferumoxytol (Feraheme, AMAG Pharmaceuticals, Lexington, MA) was delivered via a tail vein cannula and both $\mathrm{T}_{2}{ }^{*}$-weighted and $\mathrm{T}_{2}$-weighted image acquisitions were repeated 5 min later.

Image analysis. Vessel density, vessel size, $\Delta \mathrm{R}_{2}$ (CBV in small blood vessels) and $\Delta \mathrm{R}_{2}{ }^{*}(\mathrm{CBV}$ in a broad range of vessels) images were calculated using MATLAB (R2018b, The MathWorks, Natick, MA) with formulae as described in Lin et al. ${ }^{13,22}$. These transverse relaxation rate changes were then used used to investigate vessel size $\left(\Delta \mathrm{R}_{2}{ }^{*} / \Delta \mathrm{R}_{2}\right)$ and density $\left(\Delta \mathrm{R}_{2} /\left(\Delta \mathrm{R}_{2}{ }^{*}\right)^{2 / 3}\right)^{23,24}$. Regions of interest (ROI) were delineated in the ipsilateral and contralateral cortex and hippocampus and the median value for each image metric determined.

Neuromotor testing. Neuromotor function was assessed one-week post-injury using a $1 \mathrm{~m}$ long, $2 \mathrm{~cm}$ wide elevated wooden beam as previously described ${ }^{17,21}$. Briefly, rats were trained on the beam task on the day 
a

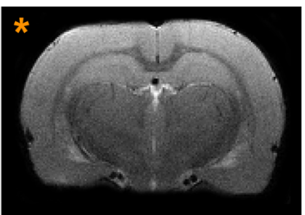

Male Sham

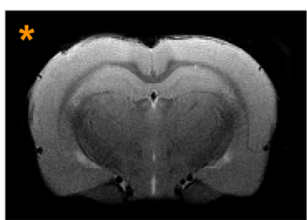

Female Sham

C
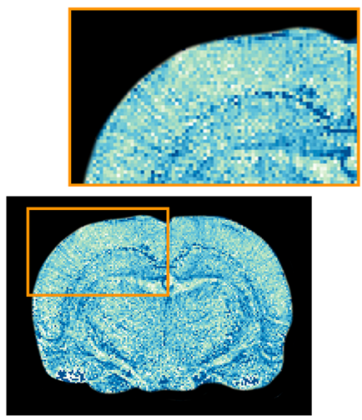

Male Sham

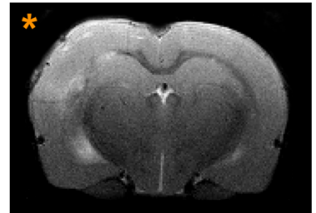

Male TBI

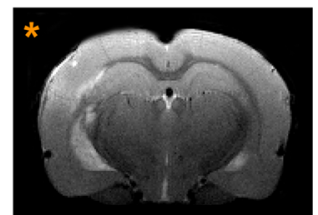

Female TBI
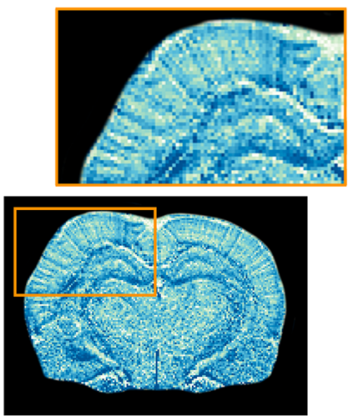

Male TBI b
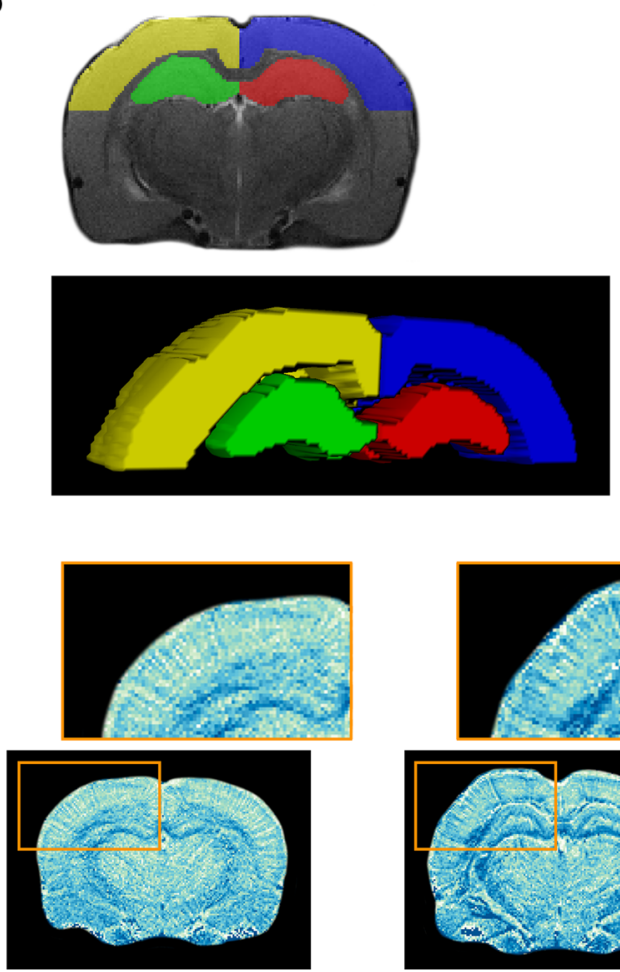

Female Sham

f Ipsilateral Hippocampus

e Contralateral Cortex

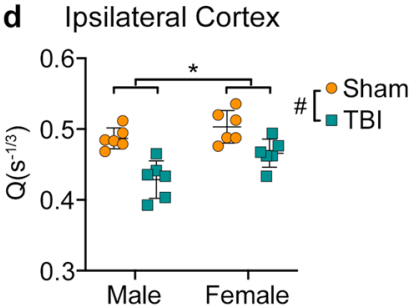

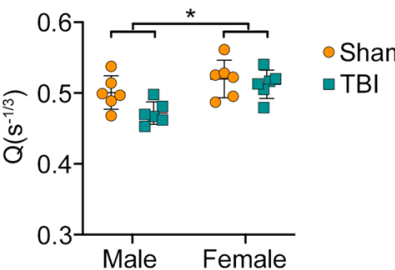

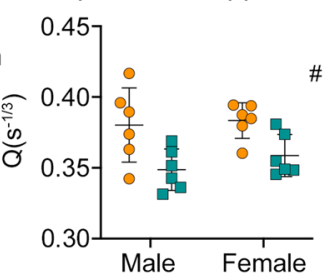

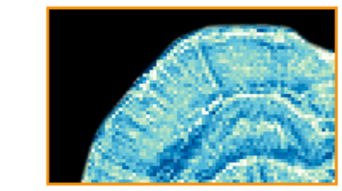

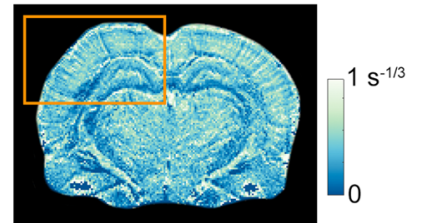

Female TBI

g Contralateral Hippocampus

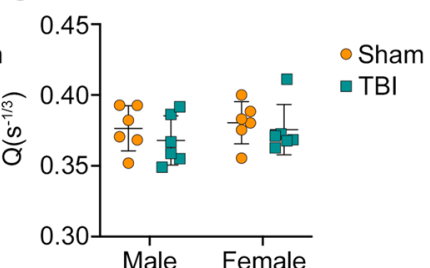

Figure 1. TBI results in decreased vascular density (Q). (a) Representative T2-weighted images from male sham, male TBI, female sham, and female TBI rats showing the extent of injury in the ipsilateral hemisphere (orange ${ }^{\star}$. (b) Example ROIs for one animal: yellow, ipsilateral cortex; green, ipsilateral hippocampus; blue, contralateral cortex; and red, contralateral hippocampus. (c) Representative Q images from male sham, male TBI, female sham and female TBI rats. The ipsilateral hippocampus and cortex are shown enlarged above (orange box). Region-based analysis revealed a significant main effect of sex in both the (d) ipsilateral and (e) contralateral cortices with females having greater $\mathrm{Q}$ than males. Furthermore, there was a significant injury effect with TBI rats having reduced Q in the ipsilateral (d) cortex and (f) hippocampus. Graphs show mean + SD. * Significant $(p<0.05)$ differences between males and females; \# significant $(p<0.05)$ differences between sham and TBI.

prior to testing. On the day of testing, rats completed ten trials with the time taken to traverse the beam, as well as the numbers of slips and falls, recorded. The maximum time allowed per trial was $60 \mathrm{~s}$, which was the value given to rats that fell.

Statistical analysis. Two-way analysis of variance (ANOVA), with sex and injury as between-subjects factors, was used for the statistical analysis of all variables SPSS 23.0 (IBM Corp., Armonk, USA). Bonferroni posthoc comparisons were completed when appropriate. Statistical significance was set at $p<0.05$.

\section{Results}

Sex and TBI affect SSCE-MRI measures. SSCE-MRI was used to study the vasculature of brain tissue $48 \mathrm{~h}$ after TBI or sham injury in male and female rats. Measures of vascular density, vessel size, $\Delta \mathrm{R}_{2}{ }^{*}$ (i.e. CBV changes in a broad range of vessel sizes), and $\Delta \mathrm{R}_{2}$ (i.e. CBV changes primarily in small vessels such as capillaries and venules) were evaluated in the cortex and hippocampus both ipsilateral and contralateral to the injury site.

Representative T2-weighted images show the effects of LFPI with evidence of edema and white matter damage (Fig. 1a). Example ROIs for one rat are shown in Fig. 1b. Representative images of vascular density are shown in Fig. 1c. Two-way ANOVA found a significant main effect of injury in the ipsilateral cortex $\left(\mathrm{F}_{1,20}=29.666\right.$, 
a

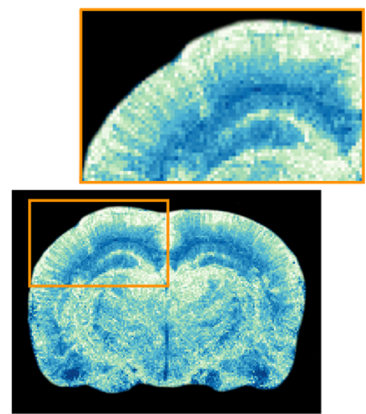

Male Sham

b Ipsilateral Cortex

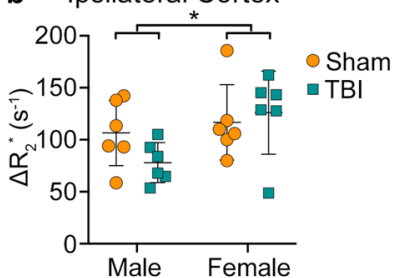

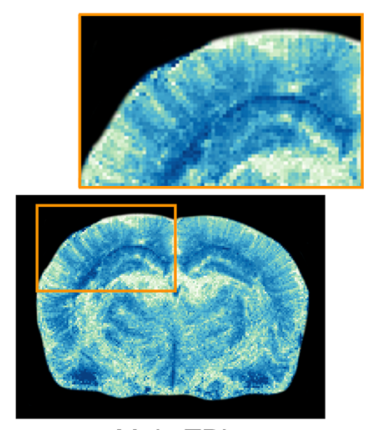

Male TBI

c Contralateral Cortex

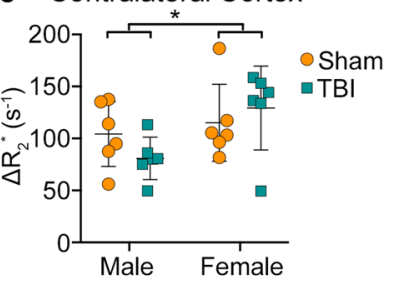

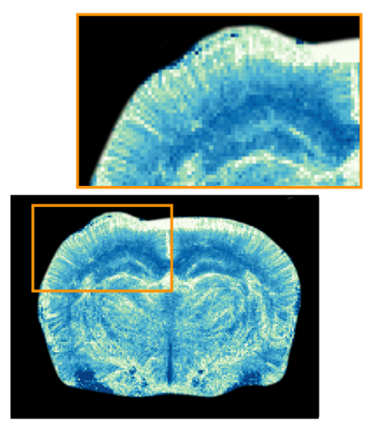

Female Sham

d Ipsilateral Hippocampus

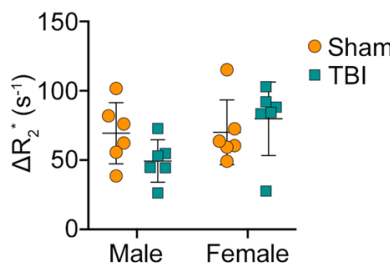

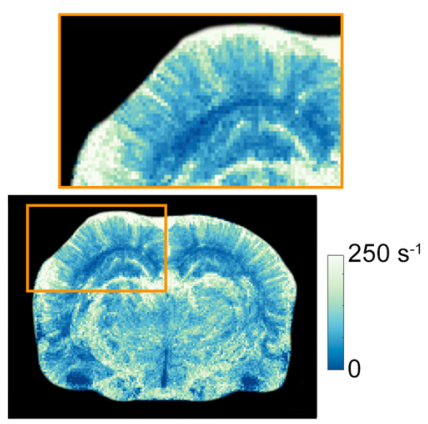

Female TBI

e Contralateral Hippocampus

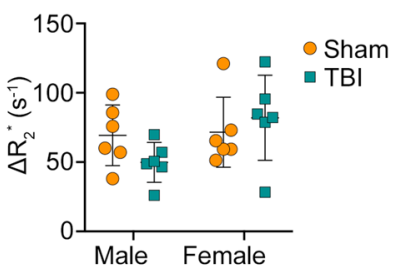

Figure 2. SSCE-MRI revealed sex differences in blood volume. (a) Representative images showing increased $\Delta \mathrm{R}_{2}{ }^{*}$ values in females compared to males. The ipsilateral hippocampus and cortex are shown enlarged above (orange box). Region-based analyses revealed that male rats have reduced blood volume, as indicated by lower $\Delta \mathrm{R}_{2}{ }^{*}$ values, in (b) ipsilateral and (c) contralateral cortices. Graphs show mean $+\mathrm{SD}$. ${ }^{\star}$ Significant $(p<0.05)$ differences between males and females.

$p<0.001$, Fig. 1d) and ipsilateral hippocampus $\left(\mathrm{F}_{1,20}=14.825, p=0.001\right.$, Fig. 1f), with TBI rats having less density. There was also a significant main effect of sex in the ipsilateral cortex $\left(\mathrm{F}_{1,20}=9.373, p=0.006\right.$, Fig. $\left.1 \mathrm{~d}\right)$ and contralateral cortex $\left(\mathrm{F}_{1,20}=11.323, p=0.003\right.$, Fig. 1e), with female rats having greater density than males. No significant differences were observed in the contralateral hippocampus (Fig. 1g).

Figure 2 shows representative images of $\Delta \mathrm{R}_{2}{ }^{*}$. Two-way ANOVA found a significant main effect of sex in the ipsilateral cortex $\left(\mathrm{F}_{1,20}=4.766, p=0.041\right.$, Fig. $\left.2 \mathrm{~b}\right)$ and contralateral cortex $\left(\mathrm{F}_{1,20}=4.820, p=0.040\right.$, Fig. $\left.2 \mathrm{c}\right)$, with female rats having greater $\Delta \mathrm{R}_{2}{ }^{*}$ than males. There were no significant differences observed for either the ipsilateral or the contralateral hippocampus (Fig. 2d,e, respectively) and no significant effect of injury was observed in any of the ROIs investigated.

Representative images of $\Delta R_{2}$ are shown in Fig. 3. There was a significant main effect of sex in the ipsilateral cortex $\left(F_{1,20}=5.762, p=0.026\right.$, Fig. 3b $)$ and contralateral cortex $\left(F_{1,20}=5.955, p=0.024\right.$, Fig. $\left.3 c\right)$, with female rats having greater $\Delta \mathrm{R}_{2}$ values than males. No significant differences were observed in either hippocampus (Fig. 1d,e) and no significant effect of injury was observed in any of the ROIs investigated.

There were so statistically significant differences found on the measure of vessel size $(p>0.050$, data not shown).

Males given TBI have worse neuromotor outcomes than females. Rats were tested on the beam task one-week post-injury to assess sensorimotor function. On the measure of traverse time, two-way ANOVA found a significant sex ${ }^{*}$ jujury interaction $\left(\mathrm{F}_{1,38}=4.086, p=0.049\right.$, Fig. $\left.4 \mathrm{a}\right)$ with the Male + TBI rats having longer traverse times compared to each of the other groups (all $p<0.005$ ). There were also significant injury $\left(\mathrm{F}_{1,38}=5.562, p=0.024\right)$ and $\operatorname{sex}\left(\mathrm{F}_{1,38}=4.442, p=0.042\right)$ effects on the measure of traverse time. On the measure of slips and falls, two-way ANOVA found significant main effects of injury $\left(\mathrm{F}_{1,38}=5.502, p=0.024\right.$, Fig. $\left.4 \mathrm{~b}\right)$ and $\operatorname{sex}\left(\mathrm{F}_{1,38}=8.296, p=0.006\right.$, Fig. $\left.4 \mathrm{~b}\right)$, with TBI and male rats having more slips.

\section{Discussion}

The majority of experimental TBI research has been conducted in males, creating a void with respect to understanding how the female brain responds to TBI. The cerebrovasculature likely plays a key role in TBI recovery as it supports tissue metabolism, edema resolution, and the clearance of debris ${ }^{4}$. Here, we employed SSCE-MRI to interrogate the microvasculature of male and female rats given experimental TBI at $48 \mathrm{~h}$ post-injury, and how SSCE-MRI measures relate to neuromotor function at 1 week-post injury. We found that TBI decreased vessel density in both males and females. However, females had superior vessel density and CBV compared to males, and female rats given a TBI had better neuromotor function compared to male TBI rats.

Our results concur with earlier studies showing an acute reduction in vascular density followed by increased vascular structure at 1 week and 2 weeks post-injury ${ }^{2,25}$. Park and colleagues used a silicone microangiography technique and immunohistochemistry in male rats also given a $3 \mathrm{~atm}$ LFPI. The authors found a significant reduction in microvasculature density, with reduced capillary numbers and diameters at $24 \mathrm{~h}$ that had recovered by 2 weeks post-injury ${ }^{26}$. Obenaus and colleagues (2017) also reported a reduced neurovascular network within male rats one day after being given a controlled cortical impact injury. Furthermore, confocal microscopy findings 
a

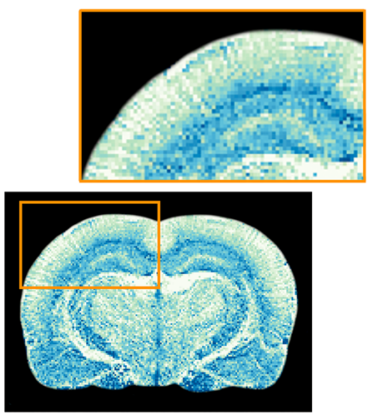

Male Sham

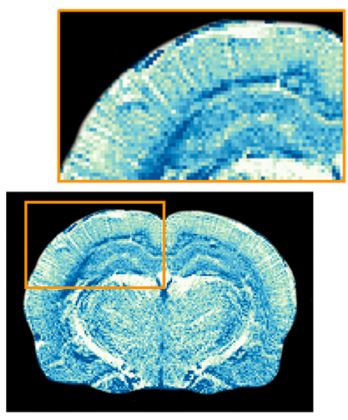

Male TB

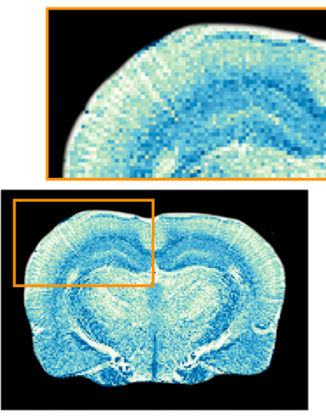

Female Sham b Ipsilateral Cortex

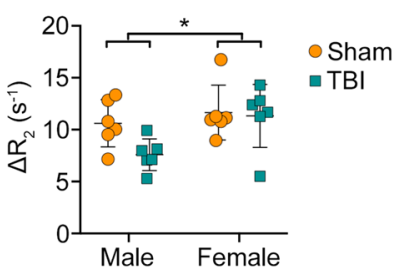

c Contralateral Cortex

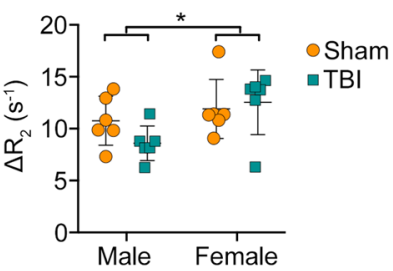

d Ipsilateral Hippocampus

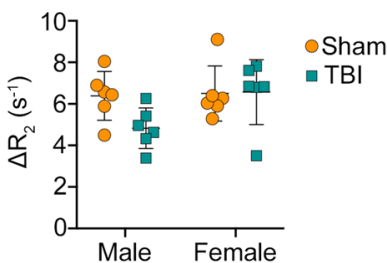

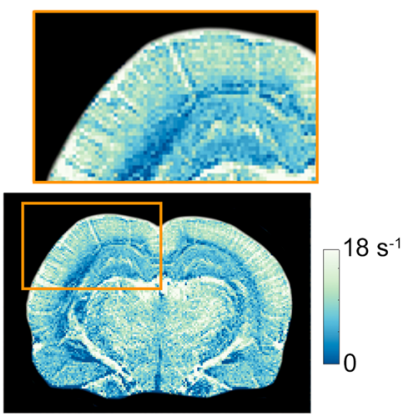

Female TBI

e Contralateral Hippocampus

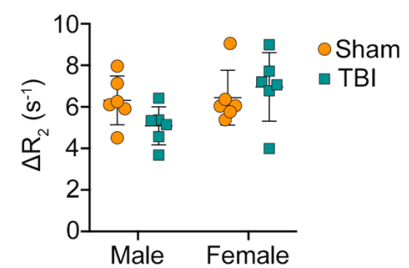

Figure 3. SSCE-MRI revealed sex differences in the blood volume of smaller vessels such as capillaries and venules. (a) Representative images showing increased $\Delta \mathrm{R}_{2}$ values in females compared to males. The ipsilateral hippocampus and cortex are shown enlarged above (orange box). Region-based analyses revealed that male rats have reduced blood volume in smaller vessels, as indicated by lower $\Delta \mathrm{R}_{2}$ values, in (b) ipsilateral and (c) contralateral cortices. Graphs show mean + SD. ${ }^{\star}$ Significant $(p<0.05)$ differences between males and females.

a

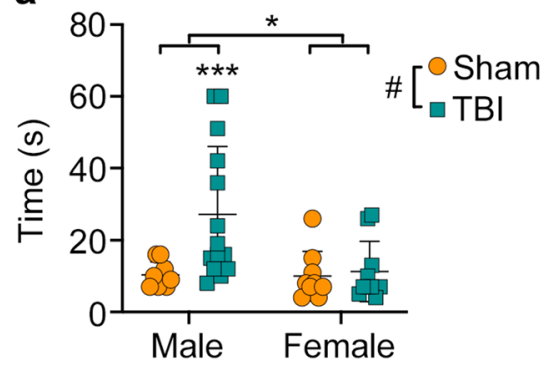

b

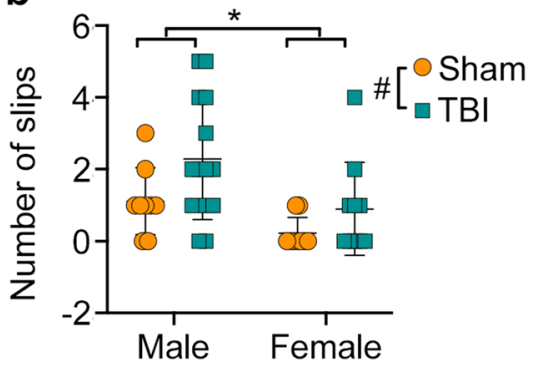

Figure 4. Behavioural testing revealed sex-specific deficits in sensorimotor function. (a) Male rats given a TBI had significantly longer traverse times than all other groups. There were also significant effects of injury and sex on traverse times. (b) Male rats and TBI cohorts made significantly more foot slips than female rats and sham cohorts, respectively. Graphs show mean $+\mathrm{SD} .{ }^{\star * *} p<0.005 ;{ }^{\star}$ Significant $(p<0.05)$ differences between males and females; "Significant $(p<0.05)$ differences between sham and TBI.

suggested that reductions in density were due to increased fragmentation of the vasculature whereby smaller branching vessels disappear and larger vessels remain ${ }^{27}$. These results would align with changes in SSCE-MRI $\Delta \mathrm{R}_{2}$ values following TBI, as they reflect CBV changes in smaller blood vessels. However, we did not find an injury effect in this study, possibly due to the timing or sensitivity of SSCE-MRI. Immonen and colleagues (2010) showed that after an initial decrease in $\Delta \mathrm{R}_{2}$ values at the site of the lesion 1 to $4 \mathrm{~h}$ post-CCI injury, $\Delta \mathrm{R}_{2}$ values had returned to sham levels by $48 \mathrm{~h}$ post-injury ${ }^{16}$. A subsequent study by the same group measured $\Delta \mathrm{R}_{2}$ values $48 \mathrm{~h}$ post-LFPI and found no significant differences in CBV between sham and TBI rats which is also consistent with the results observed here ${ }^{25}$.

We found that female rats had greater vessel density, and higher $\Delta \mathrm{R}_{2}$ and $\Delta \mathrm{R}_{2}{ }^{*}$ values in the ipsilateral and contralateral cortex when compared to their male counterparts. As disruption to the cerebral vasculature can lead to secondary injury mechanisms including hemorrhage, edema, abnormal perfusion and blood-brain barrier dysfunction ${ }^{28}$ these results may in part explain the more severe sensorimotor deficits seen in male rats. Despite conflicting evidence on gender-specific outcomes, it is clear that sex plays an important role in TBI outcomes $^{11,29}$. Notably, our sex-difference findings on the beam task are consistent with those of Wagner and colleagues (2004) who found that male rats given a controlled cortical impact TBI performed worse than females on a beam-walking task ${ }^{30}$.

There are some limitations to consider with the current study. We performed SSCE-MRI at a single acute time-point, and future studies should employ serial SSCE-MRI at acute, sub-acute, and chronic recovery times to investigate evolving changes. Our small cohort sizes for SSME-MRI coupled with our limited behavioural testing 
prevented us from conducting meaningful analyses to assess how the SSME-MRI changes relate to functional outcomes, which would be of great interest and should be explored further. We did not perform histological or immunohistochemical validation of altered vasculature and future studies could also complement SSCEMRI findings with direct measures of cellular and molecular changes related to brain vasculature. For example, vascular endothelial growth factor (VEGF) is a potent stimulator of angiogenesis and vasculogenesis, and has been found to be upregulated in experimental $\mathrm{TBI}^{28,31,32}$. Another protein that can also stimulate angiogenesis is pleiotrophin which is upregulated in the ischemic brain, and of particular relevance here, in the endothelial cells of newly formed vessels ${ }^{33}$.

In conclusion, this study used SSCE-MRI to investigate the neurovasculature of male and female rats at $48 \mathrm{~h}$ after TBI, and how these changes related to neuromotor function at 1-week post-TBI. Although TBI decreased vessel density in both sexes, female rats had greater vessel density, $\Delta \mathrm{R}_{2}$, and $\Delta \mathrm{R}_{2}^{*}$ values, and female TBI rats performed better on a neuromotor task than their male counterparts. These results suggest that cerebrovascular function, as assessed by SSCE-MRI, is superior in females and may contribute to their improved neuromotor recovery after TBI. We have also shown the potential for SSCE-MRI as a biomarker to provide insights into the cerebral microvasculature post-TBI. In practice, Ferumoxytol is a superparamagnetic iron oxide nanoparticle that was approved for the treatment of iron deficiency and anemia in patients with chronic kidney disease by the FDA. It has since been used for SSCE-MRI ${ }^{34}$, and has been proven safe and effective for clinical MRI use ${ }^{35}$. As such, SSCE-MRI could be a promising tool in detecting vascular density changes in humans with TBI. Finally, our results demonstrate the need for future translational TBI studies to incorporate both sexes, and to investigate how the neurovasculature evolves throughout TBI recovery. Such studies may provide a foundation to develop optimal interventions that improve recovery in both males and females.

\section{Data availability}

The imaging datasets analysed during the current study are available from the corresponding author on reasonable request.

Received: 18 April 2020; Accepted: 10 November 2020

Published online: 04 December 2020

\section{References}

1. Kenney, K. et al. Cerebral vascular injury in traumatic brain injury. Exp. Neurol. 275, 353-366 (2016).

2. Salehi, A. et al. Up-regulation of Wnt/ $\beta$-catenin expression is accompanied with vascular repair after traumatic brain injury. J. Cereb. Blood Flow Metab. 38, 274-289 (2018).

3. Zhang, Y., Xiong, Y., Mahmood, A., Zhang, Z. G. \& Chopp, M. Angiogenesis and functional recovery after traumatic brain injury. In Vascular Mechanisms in CNS Trauma (eds Lo, E. H. et al.) 141-156 (Springer, New York, 2013).

4. Jullienne, A. et al. Male and female mice exhibit divergent responses of the cortical vasculature to traumatic brain injury. J. Neurotrauma 35, 1646-1658 (2018).

5. Badaut, J. \& Bix, G. J. Vascular neural network phenotypic transformation after traumatic injury: Potential role in long-term sequelae. Transl. Stroke Res. 5, 394-406 (2014).

6. Xiong, Y., Mahmood, A. \& Chopp, M. Angiogenesis, neurogenesis and brain recovery of function following injury. Curr. Opin. Investig. Drugs 11, 298-308 (2010).

7. Coronado, V. G. et al. Surveillance for traumatic brain injury-related deaths-United States, 1997-2007. MMWR Surveill. Summ. 60, 1-32 (2011).

8. Gupte, R., Brooks, W., Vukas, R., Pierce, J. \& Harris, J. Sex differences in traumatic brain injury: What we know and what we should know. J. Neurotrauma 36, 3063-3091 (2019).

9. McGlade, E., Rogowska, J. \& Yurgelun-Todd, D. Sex differences in orbitofrontal connectivity in male and female veterans with TBI. Brain Imag. Behav. 9, 535-549 (2015).

10. Slewa-Younan, S., Green, A. M., Baguley, I. J., Gurka, J. A. \& Marosszeky, J. E. Sex differences in injury severity and outcome measures after traumatic brain injury. Arch. Phys. Med. Rehabil. 85, 376-379 (2004).

11. Wright, D. K., O’Brien, T. J., Shultz, S. R. \& Mychasiuk, R. Sex matters: Repetitive mild traumatic brain injury in adolescent rats. Ann. Clin. Transl. Neurol. 4, 640-654 (2017).

12. Roof, R. L. \& Hall, E. D. Gender differences in acute CNS trauma and stroke: Neuroprotective effects of estrogen and progesterone. J. Neurotrauma 17, 367-388 (2000)

13. Lin, C.-Y. et al. Dynamic changes in vascular permeability, cerebral blood volume, vascular density, and size after transient focal cerebral ischemia in rats: Evaluation with contrast-enhanced magnetic resonance imaging. J. Cereb. Blood Flow Metab. 28, 1491-1501 (2008).

14. Wu, E. X., Tang, H. \& Jensen, J. H. High-resolution MR imaging of mouse brain microvasculature using the relaxation rate shift index Q. NMR Biomed. 17, 507-512 (2004).

15. Kim, J. et al. Multiparametric characterization of response to anti-angiogenic therapy using USPIO contrast-enhanced MRI in combination with dynamic contrast-enhanced MRI. J. Magn. Reson. Imaging 47, 1589-1600 (2018).

16. Immonen, R. et al. Cerebral blood volume alterations in the perilesional areas in the rat brain after traumatic brain injury-comparison with behavioral outcome. J. Cereb. Blood Flow Metab. 30, 1318-1328 (2010).

17. Wright, D. K. et al. Behavioral, blood, and magnetic resonance imaging biomarkers of experimental mild traumatic brain injury. Sci. Rep. 6, 28713 (2016).

18. Shultz, S. R. et al. Sodium selenate reduces hyperphosphorylated tau and improves outcomes after traumatic brain injury. Brain 138, 1297-1313 (2015).

19. Brady, R. D. et al. Experimental traumatic brain injury induces bone loss in rats. J. Neurotrauma 33, 2154-2160 (2016).

20. Wright, D. K. et al. Changes in apparent fiber density and track-weighted imaging metrics in white matter following experimental traumatic brain injury. J. Neurotrauma 34, 2109-2118 (2017).

21. Wright, D. K. et al. Traumatic brain injury results in cellular, structural and runctional changes resembling motor neuron disease. Cereb. Cortex 27, 4503-4515 (2017).

22. Kiselev, V. G., Strecker, R., Ziyeh, S., Speck, O. \& Hennig, J. Vessel size imaging in humans. Magn. Reson. Med. 53, 553-563 (2005).

23. Kim, E. et al. Vasculature-specific MRI reveals differential anti-angiogenic effects of a biomimetic peptide in an orthotopic breast cancer model. Angiogenesis 18, 125-136 (2015). 
24. Kim, E. et al. Assessing breast cancer angiogenesis in vivo: Which susceptibility contrast MRI biomarkers are relevant?. Magn. Reson. Med. 70, 1106-1116 (2013).

25. Hayward, N. M. E. A. et al. Magnetic resonance imaging of regional hemodynamic and cerebrovascular recovery after lateral fluid-percussion brain injury in rats. J. Cereb. Blood Flow Metab. 31, 166-177 (2011).

26. Park, E., Bell, J. D., Siddiq, I. P. \& Baker, A. J. An analysis of regional microvascular loss and recovery following two grades of fluid percussion trauma: A role for hypoxia-inducible factors in traumatic brain injury. J. Cereb. Blood Flow Metab. 29, 575-584 (2009).

27. Obenaus, A. et al. Traumatic brain injury results in acute rarefication of the vascular network. Sci. Rep. 7, 239 (2017).

28. Salehi, A., Zhang, J. H. \& Obenaus, A. Response of the cerebral vasculature following traumatic brain injury. J. Cereb. Blood Flow Metab. 37, 2320-2339 (2017).

29. Vagnerova, K., Koerner, I. P. \& Hurn, P. D. Gender and the injured brain. Anesth. Analg. 107, 201-214 (2008).

30. Wagner, A. K. et al. Evaluation of estrous cycle stage and gender on behavioral outcome after experimental traumatic brain injury. Brain Res. 998, 113-121 (2004).

31. Morgan, R., Kreipke, C. W., Roberts, G., Bagchi, M. \& Rafols, J. A. Neovascularization following traumatic brain injury: Possible evidence for both angiogenesis and vasculogenesis. Neurol. Res. 29, 375-381 (2007).

32. Storkebaum, E. \& Carmeliet, P. VEGF: A critical player in neurodegeneration. J. Clin. Invest. 113, 14-18 (2004).

33. Yeh, H. J., He, Y. Y., Xu, J., Hsu, C. Y. \& Deuel, T. F. Upregulation of pleiotrophin gene expression in developing microvasculature, macrophages, and astrocytes after acute ischemic brain injury. J. Neurosci. 18, 3699-3707 (1998).

34. Varallyay, C. G. et al. High-resolution steady-state cerebral blood volume maps in patients with central nervous system neoplasms using Ferumoxytol, a superparamagnetic iron oxide nanoparticle. J. Cereb. Blood Flow Metab. 33, 780-786 (2013).

35. Varallyay, C. G. et al. What does the boxed warning tell us? Safe practice of using ferumoxytol as an MRI contrast agent. AJNR Am. J. Neuroradiol. 38, 1297-1302 (2017).

\section{Author contributions}

D.K.W., J.M., T.O.B., and S.R.S. made significant contributions to conception and design of the study. D.K.W., J.M., M.S., and S.R.S. acquired and analyzed the data. D.K.W., J.M., T.O.B., and S.R.S. drafted a significant portion of the manuscript or figures. All authors edited the final draft and approved the final version of the manuscript.

\section{Funding}

The authors disclosed receipt of the following financial support for the research, authorship, and/or publication of this article: This work was supported by the National Health and Medical Research Council to SRS [Grant number: 1141643; fellowship: 1159645] and to DKW [Grant number: 1174040].

\section{Competing interests}

The authors declare no competing interests.

\section{Additional information}

Correspondence and requests for materials should be addressed to S.R.S.

Reprints and permissions information is available at www.nature.com/reprints.

Publisher's note Springer Nature remains neutral with regard to jurisdictional claims in published maps and institutional affiliations.

(c) (i) Open Access This article is licensed under a Creative Commons Attribution 4.0 International cc) License, which permits use, sharing, adaptation, distribution and reproduction in any medium or format, as long as you give appropriate credit to the original author(s) and the source, provide a link to the Creative Commons licence, and indicate if changes were made. The images or other third party material in this article are included in the article's Creative Commons licence, unless indicated otherwise in a credit line to the material. If material is not included in the article's Creative Commons licence and your intended use is not permitted by statutory regulation or exceeds the permitted use, you will need to obtain permission directly from the copyright holder. To view a copy of this licence, visit http://creativecommons.org/licenses/by/4.0/.

(C) The Author(s) 2020 